\title{
Análisis financiero para la determinación de quiebra
}

Roberto López ${ }^{1}$

1 Maestría en Administración y Dirección de Empresas (MADE) Facultad de Ciencias Económicas y Empresariales de la UCA. Apdo. 69, Managua, Nicaragua.

Recibido: abril 2006 / Aceptado: mayo 2006

SE ESTUDIA LA PROBABILIDAD DE QUIEBRA DE EMPRESAS nicaragüenses en 1990, cuando surgió la banca privada tras acuerdos entre empresarios y el gobierno, que modernizaron las finanzas, pero presentaron riesgos empresariales. Se dieron restricciones legales, riesgos políticos y fragilidad de las instituciones financieras ante la banca internacional. Además, las fluctuaciones del tipo de cambio del córdoba originan cuatro riesgos: conversión, de transacciones, económico y operativo. Diferentes investigadores han desarrollado modelos de alertas financieras: J. Tobin, que desarrolló "Q de Tobin"; W. Beaver, para empresas manufactureras; M. Tamarí, un sistema de puntaje; y E. Altman desarrolló el modelo "Z de Altman", que da un índice de baja, alta o altísima probabilidad de quiebra. Se aplicó este modelo a las empresas quebradas a fines de 2000: Banco Europeo de Centroamérica (BECA), Banco Nacional de Desarrollo (BANADES), Banco de Crédito Popular (BCP), Banco del Campo (BANCAMPO), y Banco Internacional (INTEBANK).

Palabras clave: quiebra, instituciones financieras-Nicaragua, riesgo (finanzas)

\section{Introducción}

Esta investigación trata de identificar y evaluar los factores de riesgo y de fracaso empresarial. Hay pocos estudios de la Probabilidad de Quiebra de una empresa, pues los estudios pretenden encontrar los factores para el máximo rendimiento en una inversión. Sin embargo, el diagnóstico empresarial es un instrumento necesario porque, en el ámbito de los negocios, lo que aparentemente se presenta como una oportunidad de oro, puede convertirse en un barril sin fondo.

Analizar el riesgo es buscar las debilidades que hacen que una empresa vaya al fracaso e identificar sus fortalezas, para adecuar las estrategias al entorno económico. La investigación se apoya en los estados financieros, analizando cada componente de las cuentas de resultado y situación patrimonial, centrando el análisis en el porqué de los resultados y en la toma de medidas correctivas.

La dinámica del mercado y la dirección por objetivos exigen nuevos planteamientos de gestión para la competitividad, lo que demanda una cultura investigadora para adecuarse 
al entorno económico. El propósito es estimular la utilización del análisis y razones financieras de los estados financieros, para determinar las probabilidades de quiebra en las empresas.

Este estudio pretende analizar la posibilidad de señalar ese riesgo, apoyándose en herramientas utilizadas en el análisis de balances y razones financieras, para que sirvan de modelo y crear escenarios de trabajo.

Se busca identificar las variables económicas y financieras que constituyen la síntesis de la información para analizarla y definir criterios aplicando el método de Altman y Beaver, para relacionar las distintas cuentas de balance y de resultado y obtener la información de gestión. Hay poca documentación que identifique los indicadores críticos, que se puedan rastrear y que ejercen impacto negativo. Esto constituye un reto que se asume con responsabilidad, para hurgar en lo poco que se ha escrito y trasmitir la información a los interesados.

\section{Entorno económico}

En 1990 Nicaragua inició una transición política, económica y social desde la economía centralizada a la economía de libre empresa. Grupos de empresarios crearon instituciones bancarias privadas para generar ahorro interno y canalizarlo hacia la inversión privada y el consumo. La banca estatal fue gradual y deliberadamente reducida. Se restableció la Superintendencia de Bancos y, aunque no había muchos ahorrantes, nacieron muchos bancos que tuvieron que enfrentar muchos retos: modernización, desarrollo tecnológico, globalización de las finanzas, fronteras de mercado abierto y constante evolución de la teoría y práctica financiera que se enfoca hacia la estrategia. Simultáneamente, enfrentaron la normativa prudencial aplicada por la institución reguladora, al tiempo que mantenían su participación en el mercado bancario y procuraban la rentabilidad patrimonial.

Estos desafíos obligaron al sistema financiero privado a replantearse su estrategia, sacrificando el crecimiento en beneficio de la rentabilidad. En la misma línea, las tasas pagadas a los depositantes descendieron y las cobradas a los prestatarios aumentaron. Como consecuencia, el ahorro financiero nacional tiende a disminuir, lo mismo que las inversiones productivas, a causa del encarecimiento del financiamiento.

Simultáneamente, los modelos tradicionales de administración demostraron haber sido superados por la realidad, lo que generó en el mundo la llamada "modernización”, una de cuyas bases es la administración por resultados.

\section{Análisis de riesgos}

\section{Riesgos empresariales}

Son muchas las causas que pueden generar la crisis empresarial: pérdida de rentabilidad, incremento de créditos incobrables, falta de liquidez, reducción de ingresos, etc. El mayor problema se plantea cuando se realizan inversiones bursátiles en distintos rubros sin respaldo suficiente, o no se registran las operaciones en los libros contables. Otro mecanismo para trasladar dinero del ahorrante justificándolo como préstamo financiero es la creación 
de empresas financieras corporativas. Estos créditos arriesgados escapan al control del Banco Central de Nicaragua y de la Superintendencia de Bancos, porque no hay normas que faciliten la revisión de las operaciones. A ello, se suma a la inestabilidad política y social, y el encarecimiento de las tasas de interés de las fuentes financieras.

\section{Riesgos tecnológicos}

Se suele concebir la tecnología como medio para automatizar procesos. Con frecuencia se invierten recursos sin motivar ni preparar al personal para utilizarla y sin un plan estratégico de cambio. Como consecuencia, se desperdician conocimientos y se olvida que lo que conduce al adelanto es la evolución de conceptos y modelos intelectuales que protejan contra el riesgo de la tecnología misma. Asimismo, hay que calificar a los recursos humanos para la transformación de la organización.

\section{Riesgos económicos y políticos}

Los riesgos económicos tratan de evitarse mediante restricciones de orden legal: forma de organizar la administración, capital social requerido, restricciones en el orden de distribución de utilidades y obligación de encaje legal. Hay una hipótesis según la cual, la rentabilidad de la inversión y liquidez de las instituciones con restricciones, tiene menor riesgo que el de las empresas con menores restricciones financieras; pero los eventos son impredecibles.

Riesgos de orden político: ligados a la estabilidad social, del gobierno de turno y su cariz político y la clasificación de categoría de riesgo del país que tenga el organismo que quiere invertir. Nicaragua es un país de riesgo por sus condiciones políticas y económicas. Pero se han creado y modificado leyes para atraer al inversionista y dar confianza al capital. El sistema financiero nicaragüense está patrocinado por capital nacional y extranjero conforme la regulación de nuestras leyes; no obstante su composición, es permeable y débil ante la entrada de bancos internacionales y con cobertura mundial.

\section{Riesgos de tipos de cambio}

Riesgos de conversión: Las fluctuaciones del tipo de cambio de la moneda nacional originan cambios en el ingreso contable, balance general y flujos de efectivo esperados, que presentan consecuencias de distorsión en los estados financieros y los costos históricos. Además, se vuelve inconsistente con la naturaleza de las normas contables. El riesgo de conversión puede implicar una utilidad o una pérdida en una transacción internacional por la aceptación de pago nominado en la moneda del país paterno de la institución que otorga los fondos. Reduce el riesgo de conversión para los bancos, el hecho de que estén autorizados por la nueva $\operatorname{Ley}^{1}$ a efectuar operaciones en moneda nacional o extranjera y mantener activos y pasivos en moneda extranjera.

Riesgo en las transacciones: Ocurre cuando se realiza una transacción internacional que puede implicar pérdidas.

Riesgo económico: Consiste en variaciones no previstas en el tipo de cambio. Afecta las transacciones en el mercado monetario. Este riesgo puede manejarse de muchas formas ${ }^{2}$ útiles: la administración de efectivo, contratos directos en moneda sólida, contratos a futuro, etc. 
Riesgo operativo: Se define como riesgo de deficiencia en la estructura de la información. Trae como consecuencia fallas fatales en los negocios. Por lo tanto, el cuidado de entender el riesgo financiero debe ser prioridad de las estructuras administrativas.

Una las deficiencias más graves de las estructuras de las instituciones es dar mayor importancia a las crisis que a las etapas de auge lo que resta importancia al rol preventivo que deben desempeñar ${ }^{3}$.

\section{Comportamiento del riesgo}

El riesgo de quiebra es intrínseco a la empresa y al país, porque la especulación del capital muchas veces supera el monto del comercio y las inversiones mundiales. El comportamiento del riesgo en los mercados financieros está sujeto a la volatilidad, con grandes consecuencias para el patrimonio de personas y empresas, lo que ha provocado quiebras y crisis económicas.

El comportamiento de una serie de variables que convergen en el sistema financiero nacional e internacional, tiene que ser seguido constantemente para saber cómo operan los mercados y ver de qué manera intervienen las tasas de interés y tipos de cambio.

Para ilustrar este tema, se utiliza un ejemplo que brinda un documento del modulo de Finanzas Internacionales ${ }^{4}$ :

1. Un prestatario que obtiene un financiamiento denominado en dólares estadounidenses a una tasa de interés flotante ligada a la LIBOR (London Interbank Offer Rate), puede incurrir en costos no previstos si aumenta dicha tasa.

2. Una compañía que contrata un préstamo en GBP (Libra esterlina), se expone a un mayor adeudo si el tipo de cambio córdobas-dólares estadounidenses se eleva, y el tipo de cambio de dólares-libra esterlina aumenta.

3. Un exportador con cuentas por cobrar en marcos alemanes (DEM), puede incurrir en una drástica reducción de sus utilidades, si el tipo de cambio marcos alemanes-dólares estadounidenses se eleva.

En consecuencia, analizar el comportamiento del riesgo implica tener herramientas para tomar la decisión de acuerdo a los objetivos de las instituciones y estas herramientas deben ser analíticas y útiles. Las instituciones deben comprender que lo que buscan los oferentes de capital es la liquidez de la empresa. Los acreedores indagan por medio de análisis profundos la capacidad de pago y la rentabilidad de sus proyectos. Por otra parte, las instituciones reguladoras desean conocer la tasa de rendimiento que obtiene una empresa sobre sus activos, y la proporción de fondos no relacionados con el capital utilizado en el negocio.

Para estos fines señalados, se utilizarán cuatro grupos de indicadores financieros que analizan razones de liquidez, razones de deuda, razones de rentabilidad y razones de cobertura, evaluando la comparación de las cantidades brutas y las razones de un balance general de un año de las instituciones financieras que estaban activas a finales del año 1995; también se aplicarán los indicadores recomendados por el economista E. Altman y el profesor William Beaver, quienes realizaron estudios particulares y aplicaron técnicas 
diferentes a las tradicionales, que ayudaron a encontrar señales y advertencias de riesgos financieros.

El riesgo es de clasificación cualitativa y el uso de análisis cuantitativos facilita el mejor juicio cualitativo, siempre que la clasificación de riesgo sea una opinión, porque los factores fundamentales del negocio cambian con el tiempo y las administraciones reaccionan de forma diversa.

\section{Modelos de alerta financiera}

\section{Q de Tobin}

El economista James Tobin desarrolló un modelo para conocer el momento de tomar la decisión de invertir o desvincularse de la actividad. Este método, llamado "Q de Tobin", se usa en las empresas que cotizan en Bolsa de Valores. Es el producto de dividir el valor de la empresa en función de la rentabilidad esperada, entre el costo de reponer sus activos reales.

La comprensión de esta operación está dada por el resultado y para ello Tobin definió que: Si Q es mayor que 1, la empresa está valorada por encima del precio de mercado. En consecuencia la empresa es rentable y da confianza para seguir en el negocio.

Si Q es menor que 1, la inversión no está produciendo la rentabilidad esperada y deben aplicarse medidas correctivas o finalizar la inversión.

Si Q es igual a 1, la inversión está en su punto de equilibrio, lo que significa que la rentabilidad es dudosa. Se debe vigilar atentamente el comportamiento de los indicadores para enderezar el rumbo de la empresa.

\section{William Beaver}

Realizó su análisis sobre una muestra de empresas manufactureras, algunas de las cuales quebraron y otras se mantuvieron activias. Analizó el desempeño de 30 índices financieros y clasificó los mejores como predictores de quiebra en el siguiente orden: 1) relación flujo de efectivo a deuda total; 2) relación utilidad neta a activos totales; 3) relación deuda total a activos totales; 4) relación capital de trabajo neto a activos totales y; 5) la razón circulante; asignando a cada uno un factor-valor para distinguir los resultados y evaluar la marcha de la empresa.

\begin{tabular}{|c|c|c|c|}
\hline Flujo de caja/deuda total & $=$ & $\begin{array}{l}\text { entre } 0.45 \text { a } 0.5 \\
\text { entre } 0.15 \text { a }-0.15\end{array}$ & $\begin{array}{l}\text { Empresas buena } \\
\text { Empresas malas }\end{array}$ \\
\hline Utilidad neta/activo total & $=$ & $\begin{array}{l}\text { entre } 0.08 \text { a } 0.09 \\
\text { entre } 0.02 \mathrm{a}-0.02\end{array}$ & $\begin{array}{l}\text { No quiebra } \\
\text { Quiebra }\end{array}$ \\
\hline Deuda total/activo total & $=$ & $\begin{array}{l}\text { entre } 0.37 \text { a } 0.45 \\
\text { entre } 0.52 \text { a } 0.81\end{array}$ & $\begin{array}{l}\text { Empresa buena } \\
\text { Quiebra }\end{array}$ \\
\hline Capital de trabajo neto/activo total & $=$ & $\begin{array}{l}\text { entre } 0.36 \text { a } 0.42 \\
\text { entre } 0.30 \text { a } 0.06\end{array}$ & $\begin{array}{l}\text { Empresa buena } \\
\text { Quiebra }\end{array}$ \\
\hline
\end{tabular}


$\begin{array}{llll}\text { Activo circulante/pasivo circulante }=\quad \begin{array}{ll}\text { entre } 3.2 \text { a } 3.4 \\ \text { entre } 2.5 \text { a } 2.0\end{array} & \text { Quiebra }\end{array}$

Enfatiza el profesor Beaver que su índice de flujo de caja a deuda total predice la quiebra con un porcentaje de certeza del $90 \%$ y el índice circulante vaticina con certeza del $80 \%$.

\section{Tamari}

Utilizó un sistema de puntajes, trabajando con seis índices financieros para pronosticar la quiebra: razón de endeudamiento; razón circulante; razón cobertura de deuda; capacidad financiera; razón de conversión de activos fijos; y reposición de capital de trabajo.

El total de puntos asignados fue de 100 y a dos de los índices los valoró en 25 a cada uno, otro $20 \mathrm{y}$ al resto en 10 puntos cada uno, utilizando criterio de opiniones y sin ninguna base estadística. Al índice circulante le asignó un máximo de 20 puntos, utilizando la escala siguiente:

Valor índice
$>2$
1.5 a 2.0
1.1 a 1.5
0.9 a 1.1
$<0.9$
tos se utilizan para fo
categoría:
< 30 puntos
de 30 a 60 puntos
$>60$ puntos

\section{Puntos}

20

15

10

5

0

Estos puntos se utilizan para formar los "Indices de Riesgo". La sumatoria resultante se divide por categoría:
$<30$ puntos
Probablemente va a la quiebra
Existe un riesgo marginal
La empresa es buena

Esta herramienta puede ser utilizada para fines crediticios, apoyándose sobre una base estadística para fortalecer los resultados y respaldar las opiniones y observaciones.

\section{Edward Altman}

Desarrolló el método del análisis discriminante que prueba la correlación entre índices y acoge los que obtienen mayor valor discriminante, llamado "Valor de Z". Es útil porque combina muchas características para tener un sólo valor con el que clasificar las alternativas de valor alto, bajo o intermedio.

El valor de $\mathrm{Z}$ alto caracteriza a una empresa financieramente saludable; un valor por debajo del resultado del Valor de Z bajo, advierte que la empresa está en peligro de quiebra. El valor de $\mathrm{Z}$ intermedio, es un resultado de connotaciones preocupantes porque es una empresa de futuro incierto.

A medida que profundizó en los estudios, fue desarrollando nuevas versiones del Valor de $\mathrm{Z}$ hasta obtener el Valor de $\mathrm{Z}_{2}$, luego de recibir críticas de analistas que alegaban su inaplicabilidad a las empresas comerciales y de servicio. 
La primera versión del Valor de $\mathrm{Z}$ fue la siguiente:

$\mathrm{Z}=1.2 * \mathrm{X}_{1}+1.4 * \mathrm{X}_{2}+3.3 * \mathrm{X}_{3}+0.6 * \mathrm{X}_{4}+0.99 * \mathrm{X}_{5}$

Las $\left(\mathrm{X}_{\mathrm{s}}\right)$ son los índices que conforman el Valor $\mathrm{Z}$ y son como sigue:

$\mathrm{X}_{1}=$ Capital de trabajo neto/activo total

$\mathrm{X}_{2}=$ Ganancias retenidas acumuladas/activo total

$\mathrm{X}_{3}=$ Ganancias antes de intereses e impuestos/activo total

$\mathrm{X}_{4}=$ Valor de mercado del patrimonio/pasivo total

$\mathrm{X}_{5}=$ Ventas/Activo total

La valoración de referencia para el análisis se clasifica así:
$\mathrm{Z}>=2.99$
Baja probabilidad de quiebra
$\mathrm{Z}<=1.81$
$1.81>\mathrm{Z}<2.99$
Alta probabilidad de quiebra
Zona gris

El grado de importancia que tienen estos índices en relación a las funciones se ubica de la siguiente manera:

Indice
$\mathrm{X}_{3}$
$\mathrm{X}_{5}$
$\mathrm{X}_{4}$
$\mathrm{X}_{2}$
$\mathrm{X}_{1}$

Posición
$1_{\text {ro. }}$
$2_{\text {do. }}$
$3_{\text {ro. }}$
$4_{\text {to. }}$
$5_{\text {to. }}$

El modelo se puede aplicar a un grupo de empresas o a una en particular. Para un grupo, se calcula cada índice señalado en el modelo para cada empresa y luego se saca el valor promedio del grupo y se sustituye en la función discriminante. En el caso de una empresa, el resultado es único para obtener el Valor de Z. Después de calcular el Valor de Z, se compara con los parámetros de referencia para ubicar el lugar de predicción en que califica.

\section{Valor de $Z_{1}$}

Después de esta primera versión siguieron dos más, mejoradas: $\mathrm{Z}_{1}$ y $\mathrm{Z}_{2}$. En el Valor de $\mathrm{Z}_{1}$ se sustituye el numerador del índice $\mathrm{X}_{4}$, que en el Valor de $\mathrm{Z}$ tiene como numerador el valor de mercado del patrimonio y en el Valor de $\mathrm{Z}_{1}$ pasa a ser el valor contable del patrimonio, porque según los críticos, para las empresas no cotizadas en el mercado es difícil precisar su valor. Entonces, $\mathrm{X}_{4}$ en la versión $\mathrm{Z}_{1}$ quedó registrada así:

$\mathrm{X}_{4}=$ Valor contable del Patrimonio/Pasivo Total

También cambia el coeficiente de la función discriminante y queda de la siguiente manera:

$$
\mathrm{Z}_{1}=0.717 * \mathrm{X}_{1}+0.847 * \mathrm{X}_{2}+3.107 * \mathrm{X}_{3}+0.42 * \mathrm{X}_{4}+0.998 * \mathrm{X}_{5}
$$

y los parámetros de referencia cambian también:

$$
\begin{array}{ll}
\mathrm{Z}_{1}>=2.90: & \text { Baja probabilidad de quiebra } \\
\mathrm{Z}_{2}<=1.23: & \text { Alta probabilidad de quiebra } \\
\mathrm{Z}_{3}<\mathrm{Z} 1<2.90: & \text { Zona gris. }
\end{array}
$$

Después de esta versión, el cuestionamiento continuó, porque aún no satisfacía el uso en 
otro tipo de empresas comerciales y de servicio que eran cotizadas o no en la Bolsa de Valores .

\section{Valor de $Z_{2}$}

La modificación realizada al Valor de $\mathrm{Z}_{1}$ consistió en la eliminación de $\mathrm{X}_{5}$, manteniendo el resto de los índices. El índice de $\mathrm{X}_{5}$, según los críticos, variaba significativamente el resultado del método y ocasionaba distorsión en el dato final, lo cual generaba interpretaciones equivocadas.

La función discriminante, luego de la modificación, se formula de esta manera:

$$
\mathrm{Z}_{2}=6.56 * \mathrm{X}_{1}+3.26 * \mathrm{X}_{2}+6.72 * \mathrm{X}_{3}+1.05 * \mathrm{X}_{4}
$$

Por supuesto que también las referencias sufren cambios y limitan nuevos parámetros, conservando la forma de calcular la fórmula y valores de cada índice para encontrar el Valor de $\mathrm{Z}_{2}$ y la interpretación se hace bajo el siguiente perfil:

$$
\begin{array}{ll}
\mathrm{Z}_{2}>=2.60: & \text { Baja probabilidad de quiebra } \\
\mathrm{Z}_{2}<=1.10: & \text { Alta probabilidad de quiebra } \\
1.10<\mathrm{Z}_{2}<2.60: & \text { Zona gris }
\end{array}
$$

\section{Significado de la resultante de Valores de Z}

La interpretación de los resultados del Valor de $\mathrm{Z}_{2}$, deber reforzarse con otras herramientas para darle mayor fortaleza y confianza al análisis e interpretación de los resultados.

Una inclinación de $\mathrm{Z}_{2}$ con valores mayores que el parámetro de clasificación de baja probabilidad de quiebra indica que la empresa tiene una bonanza que le ayuda a enfrentar problemas coyunturales e inesperados.

La tendencia por debajo de la probabilidad de quiebra, de alguna manera implica que hay riesgo y que la empresa tiene alta probabilidad de riesgo de quiebra y que, si no se toman las medidas gerenciales a tiempo, lo más seguro es que la empresa quiebre.

La zona gris corresponde a la situación en la cual los valores marcan una tendencia intermedia y es cuando existe la posibilidad de que lo impredecible ocurra, en tanto es importante considerar la zona gris como una señal de advertencia y buscar nuevas estrategias independientemente de la versión del modelo de $\mathrm{Z}$ que se utilice.

El uso más frecuente de estos modelos se ha extendido a los análisis de crédito, análisis de inversiones, análisis de auditorías, análisis legal, análisis para fusión de empresas, análisis de costo-beneficio y control e informe de gestión. Prácticamente, el examen está ligado a todo el ambiente y entorno interno y externo, donde es posible tener una visión del panorama, coadyuvando a la toma de decisiones objetivas.

\section{Aplicación de los índices de riesgo}

Los modelos e índices señalados se utilizarán en los Estados Financieros cortados al año 1995 de las instituciones financieras que al año 2000 han desaparecido del mercado 
financiero (BANADES, BECA, BANCAMPO, E INTERBANK).

El motivo de cierre de estas instituciones es conocido por el pueblo, pero se sintetizan las causas aparentes.

\section{Banco Europeo Centroamericano (Beca)}

El principal funcionario del BECA lanzó su candidatura para la presidencia de la república, lo que aceleró el cuestionamiento de la sanidad financiera del banco, porque acumulaba pérdidas desde los años anteriores a 1995 y la Superintendencia de Bancos preguntó sobre eso y sobre por qué no se había efectuado la reunión de la Asamblea General de Accionistas, como lo manda la Ley de Superintendencia de Bancos. Sin embargo, los funcionarios de la institución manifestaron que la pérdida reflejada en los estados financieros era consecuencia de la expansión de los servicios al resto del país. No obstante, los argumentos, cualesquiera que estos sean, el BECA no escapó a la falta de cuidado, visión y supervisión de las variables de riesgos estratégicos y operativos, clave para identificar los factores de transformación del círculo financiero y capacidad consistente y adecuada para mantener y ampliar el servicio sin perjuicio económico.

Los datos financieros obtenidos del informe de la Superintendencia de Bancos a 31 de Diciembre de 1995 permiten mostrar los cálculos con los modelos de Beaver y Altman.

Se realizó un resumen de las aplicaciones de Altman a los estados financieros y se obtuvo un resultado final negativo de $-0.07 \mathrm{y}$ el indicador de $Z_{2}<=1.10$ señala que, por debajo de ese valor, una empresa está en alta probabilidad de quiebra. Pero no existe la alta probabilidad de quiebra, sino la quiebra. En consecuencia, las autoridades superiores de la Superintendencia de Bancos tomaron una decisión técnica al determinar que el BECA tenía una situación de iliquidez y los socios no mostraron interés en reponer el patrimonio por pérdidas acumuladas, por lo que se hizo necesario intervenir y garantizar los depósitos del público.

La fórmula numérica y la gráfica evidencia la crisis financiera institucional:

$\mathrm{XI}=$ Capital de trabajo neto/activo total (4)

$\mathrm{X} 2$ = Ganacia retenida acumulada/activo total (3)

$\mathrm{X} 3$ = Ganancia antes de interes e impuesto/ activo total (1)

$\mathrm{X} 4=$ Valor de capital patrimonial/pasivo total

$\mathrm{Z} 2=6.56 \mathrm{X} 1+3.26 \mathrm{X} 2+6.72 \mathrm{X} 3+1.05 \mathrm{X} 4$

La ilustración gráfica muestra la imagen del comportamiento de los valores de Xs y la tendencia logarítmica y polinómica que convergen hacia una misma dirección, buscando el resultado de $\mathrm{Z}$ de Altman. 


\section{BECA: ìndice gràfico de ALTMAN}

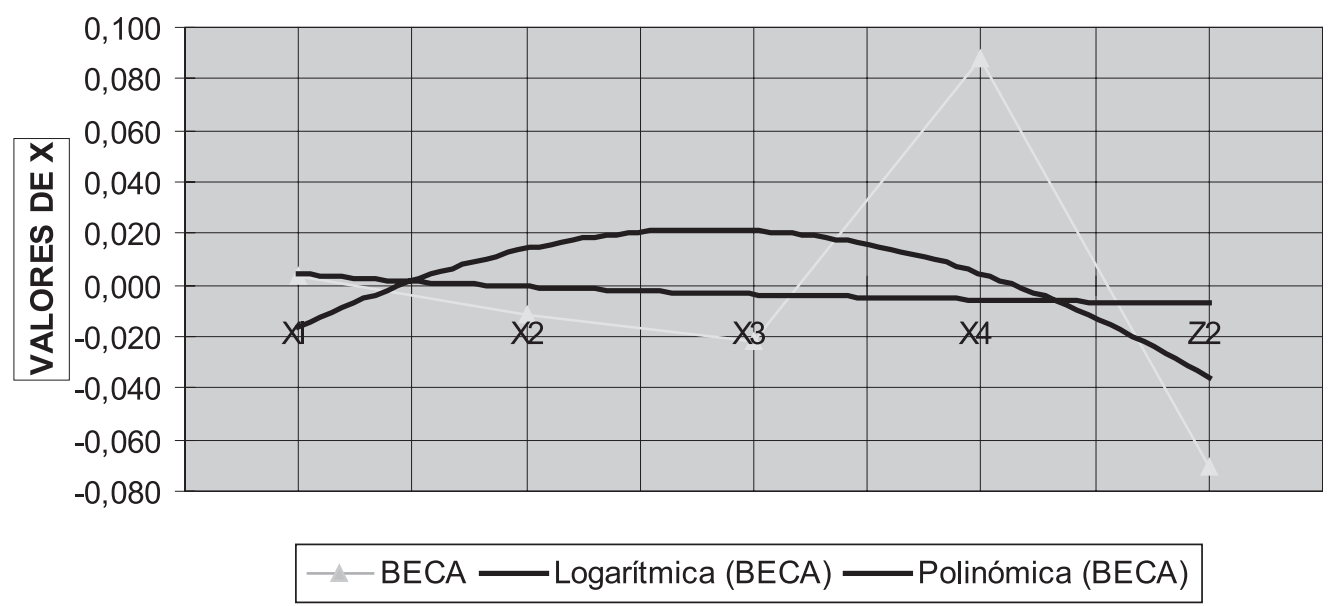

Continuando el análisis con el método de Beaver, siempre sobre la situación del BECA, se tomó en cuenta su primer índice de predicción: flujo de caja a deuda total, el cual refiere que tiene una certeza del 90\% para anunciar la quiebra. El resultado de esta operación es 0.024 , valor que está dentro de los parámetros que le asignó el patrocinador de esta teoría: flujo de caja a deuda total $=$ entre 0.15 a -0.15 son empresas malas.

\section{Banco Nacional de Desarrollo (BANADES)}

En 1995, BANADES fue sometido al análisis de las autoridades del Banco Central de Nicaragua para discutir su liquidación o disolución, pues estaba quebrado por la irresponsabilidad de sus autoridades el manejo de la política crediticia, al entregar préstamos a sectores no contemplados en los planes del gobierno y sin respaldo suficiente. Ya en 1992, la cartera del BANADES había sido saneada por el gobierno.

La incapacidad de conseguir que el gobierno central continuara con el flujo de efectivo y el no poder controlar los desajustes en el flujo financiero, hizo que la liquidación fuera la única alternativa de solución, lo cual resulta certificado con los resultados que aporta el índice Z de Altman y reforzado por el gráfico y las razones financieras de Beaver.

Edward Altman

$\mathrm{X}_{\mathrm{I}}=$ Capital de trabajo neto/ activo total (4)

$\mathrm{X}_{2}=$ Ganancia retenida acumulada/ activo total (3)

$\mathrm{X}_{3}=$ Ganancia ante interes e impuesto/ activo total (1)

$\mathrm{X}_{4}=$ Valor de capital patrimonial/ pasivo total (2)

$\mathrm{Z}_{2}=6.56 \mathrm{X} 1+3.26 \mathrm{X} 2+6.72 \mathrm{X} 3+1.05 \mathrm{X} 4$

$\mathrm{Z}_{2}<1.10>(0.685)$, lo cual equivale a demostrar que el BANADES fue quebrado. 


\section{W, Beaver}

Flujo de caja/deuda total

Utilidad neta/activo total

Deuda total/activo total

Capital de trabajo neto/activo total

Activo circulante/pasivo circulante

Cobertura del servicio de la deuda

Utilidad antes de interes e impuestos/interés + (pago principal/1-

tasa de impuesto)

Deuda total/capital contable

Razón de endeudamiento

Flujo de efectivo/deuda a largo plazo

Razón circulante

Activo circulante/Pasivo circulante

Activo circulante - Inventarios/Pasivo circulante

BANADES: modelo gràfico de $Z$ de ALTMAN

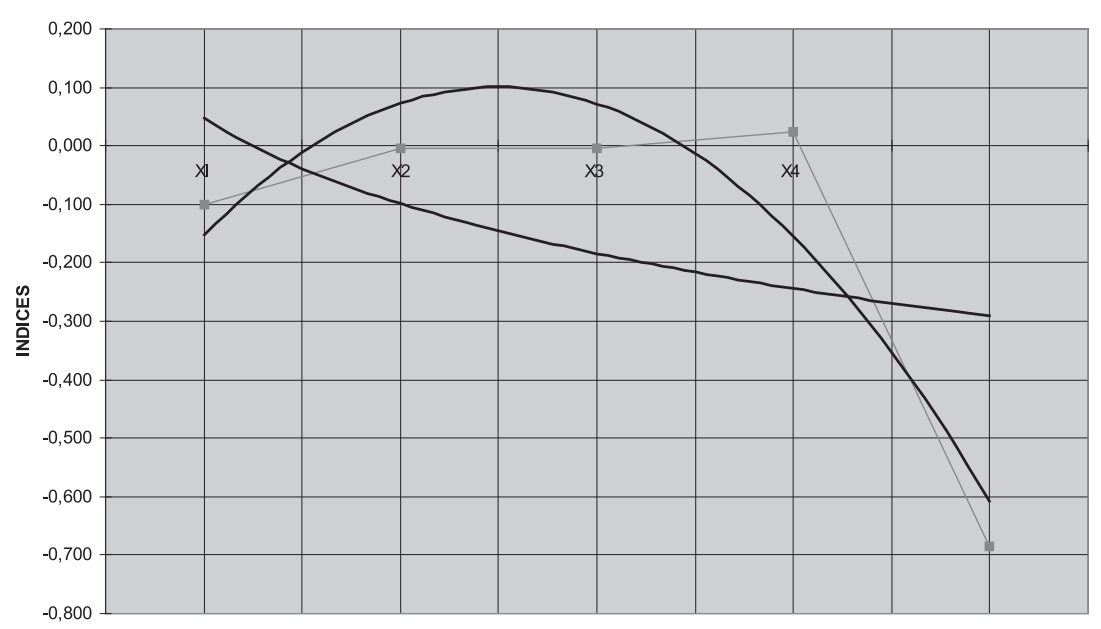

- BANADES —Polinómica (BANADES) —-Logaritmica (BANADES)

\section{Banco de Crédito Popular (BCP)}

Fue una institución del estado orientada a financiar al pequeño comerciante y hacer préstamos a trabajadores. No escapó al factor de política y estrategia de los organismos internacionales que exigieron la privatización de la banca estatal. Pero aún con el respaldo económico del gobierno central, el BCP no escapó a los malos manejos administrativos y financieros. Tuvo limitaciones y restricciones para acceder al crédito financiero, lo que finalmente llevó a su liquidación. No se conocen mecanismos para sanear la institución cuando su capital contable necesita reponerse al menos treinta veces su valor para pagar la deuda, o que el flujo de caja genere lo necesario para amortizar la deuda a largo plazo. 
El índice de Z de Altman aplicado a los estados financieros, refleja una cifra negativa mucho más elevada que la del BANADES, como puede observarse en el cuadro y gráfico siguiente:

Edward Altman

$\mathrm{XI}=$ Capital de trabajo neto/activo total (4)

$\mathrm{X} 2$ = Ganacia retenida acumulada/activo total (3)

$\mathrm{X} 3=$ Ganancia antes de impuesto e interes/activo total (1)

$\mathrm{X} 4=$ Valor capital patrimonial/Pasivo total

$\mathrm{Z} 2=6.56 \mathrm{X} 1+3.26 \mathrm{X} 2+6.72 \mathrm{X} 3+1.05 \mathrm{X} 4$

W, Beaver

Flujo de caja/deuda total

Utilidad neta/activo total

Deuda total/activo total

Capital de trabajo neto/activo total

Activo circulante/pasivo circulante

1.152

\section{BCP: indice grâfico de ALTMAN}

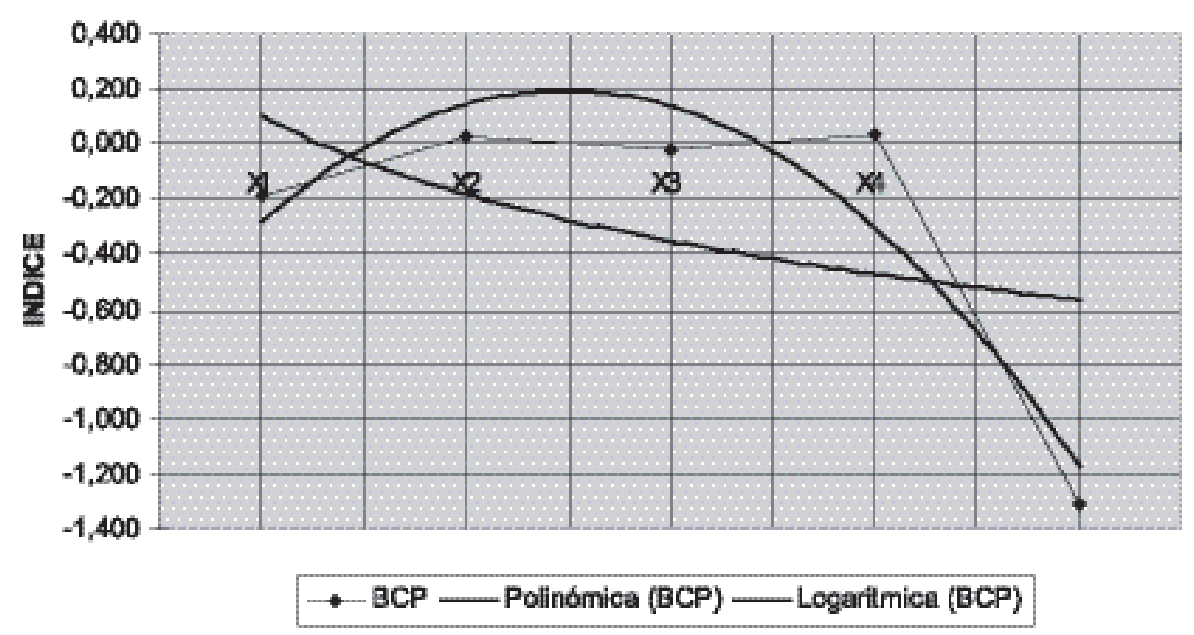

\section{Banco del Campo (BANCAMPO) (BANCO SUR)}

Uno de los primeros bancos privados que orientó su política de crédito hacia el sector rural fue el BANCAMPO. Su junta directiva, en su mayoría, estaba conformada por miembros de asociaciones agrícolas y ganaderas para atender al sector campesino que no financiaba la banca estatal ni la privada. Pero ante la falta de recursos externos que posibilitaran la asistencia al sector, la institución fue reestructurada en la composición de su directiva y la inyección de capital extranjero abrió las puertas a nuevos miembros accionistas que pasaron a ser dueños mayoritarios, de manera que se transformó en una nueva instancia legal: Banco Sur. 
En 1999, esta empresa fue intervenida por la Superintendencia de Bancos, que solicitó la corrección y aportación de capital fresco al descubrir que los funcionarios directivos estaban involucrados en una supuesta falsificación de certificados negociables de inversión (CENI's) y negociación de letras de cambio de empresas independientes del banco, pero que BANCOSUR avalaba y que luego traficaban en el mercado financiero nacional para obtener recursos líquidos al margen de los registros contables y de la sanidad financiera de la institución lo cual provocó la parálisis financiera.

La crisis financiera de BANCAMPO se configura en sus estados financieros y permiten diagnosticar los aspectos de solvencia y rentabilidad, según sus documentos cortados al año 1995, aplicando los modelos y técnicas estadísticas de predicción de fracaso.

La crisis financiera de BANCAMPO se configura en sus estados financieros y permiten diagnosticar los aspectos de solvencia y rentabilidad, según sus documentos cortados al año 1995, aplicando los modelos y técnicas estadísticas de predicción de fracaso.

Edward Altman

$\mathrm{XI}=$ Capital de trabajo neto/activo total (4)

$\mathrm{X} 2$ = Ganancia retenida acumulada/activo total (3)

$\mathrm{X} 3$ = Ganancia antes de interes e impuesto/activo total (1)

$\mathrm{X} 4$ = Valor de capital patrimonial /pasivo total

$\mathrm{Z} 2=6.56 \mathrm{X} 1+3.26 \mathrm{X} 2+6.72 \mathrm{X} 3+1.05 \mathrm{X} 4$

W, Beaver

Flujo de caja/deuda total

Utilidad neta/activo total

Deuda total/activo total

Capital de trabajo neto/activo total

Activo circulante/pasivo circulante

Cobertura del servicio de la deuda

Utilidad antes de interes e impuesto/interés + (Pago principal/1-Tasa

de impuesto)

Deuda total/Capital contable

Razón de endeudamiento

Flujo de efectivo/Deuda a largo plazo

Razón circulante

Activo circulante/Pasivo circulante

Activo circulante - Inventarios/Pasivo circulante 
BANCAMPO: ìndice gràfico de ALTMAN

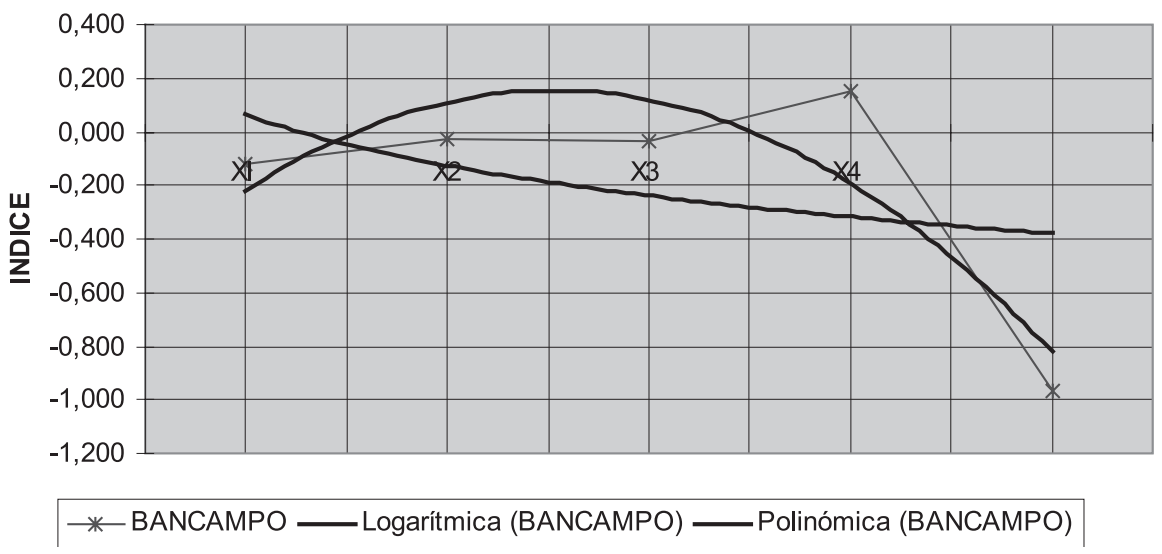

Respecto a la Z de Altman, su valor es concluyente en demostrar la alta probabilidad de quiebra al ubicarse por debajo del parámetro que señala el riesgo. También es inobjetable la certeza de predicción de quiebra del profesor Beaver cuando califica a las empresas que están ubicadas dentro del parámetro de 0.15 a -0.15 en la relación flujo de caja a deuda total, como empresas malas y asegura que este índice tiene una confianza de certeza del $90 \%$.

\section{Banco Intercontinental (INTERBANK)}

Al 31 de diciembre de 1995, los estados financieros de esta institución no presentaban pérdidas en el ejercicio ni acumuladas, aunque la Z de Altman lo ubica en el parámetro de alta probabilidad de quiebra y el parámetro del profesor Beaver lo ubica como empresa mala. Pero tenía perspectivas de corregir y adecuar las estrategias porque el resultado de los índices aplicados son todos positivos, aunque con resultados de una empresa en riesgo de quiebra. Esta institución se financia y da asistencia financiera con los depósitos de los ahorrantes, de manera que su capital de trabajo es totalmente negativo situando al banco en una situación de insolvencia financiera y a su vez obstaculizando su desarrollo.

Aplicando el análisis discriminante de Altman y los predictores de quiebra de Beaver a los estados financieros de 1995, junto con la impresión gráfica se tienen los siguientes resultados:

Edward Altman

$\mathrm{XI}=$ Capital de trabajo neto/activo total (4)

$\mathrm{X} 2$ = Ganancia retenida acumulada/activo total (3)

$\mathrm{X} 3=$ Ganancia antes de interes e impuesto/activo total (1)

$\mathrm{X} 4=$ Valor de capital patrimonial/pasivo total $(2)$

0.008

$\mathrm{Z} 2=6.56 \mathrm{X} 1+3.26 \mathrm{X} 2+6.72 \mathrm{X} 3+1.05 \mathrm{X} 4$

0.066 
W, Beaver

Flujo de caja/Deuda total

0.019

Utilidad neta/Activo total

Deuda total/Activo total

Capital de trabajo neto/Activo total

Activo circulante/Pasivo circulante

Cobertura del servicio de la deuda

Utilidad antes de interes e impuesto/Interés + (Pago principal/1-Tasa

de impuesto)

Deuda total/Capital contable

Razón de endeudamiento

Flujo de efectivo/Deuda a largo plazo

Razón circulante

Activo circulante/Pasivo circulante

Activo Circulante - Inventarios/Pasivo circulante

INTERBANK: ìndice gràfico de ALTMAN

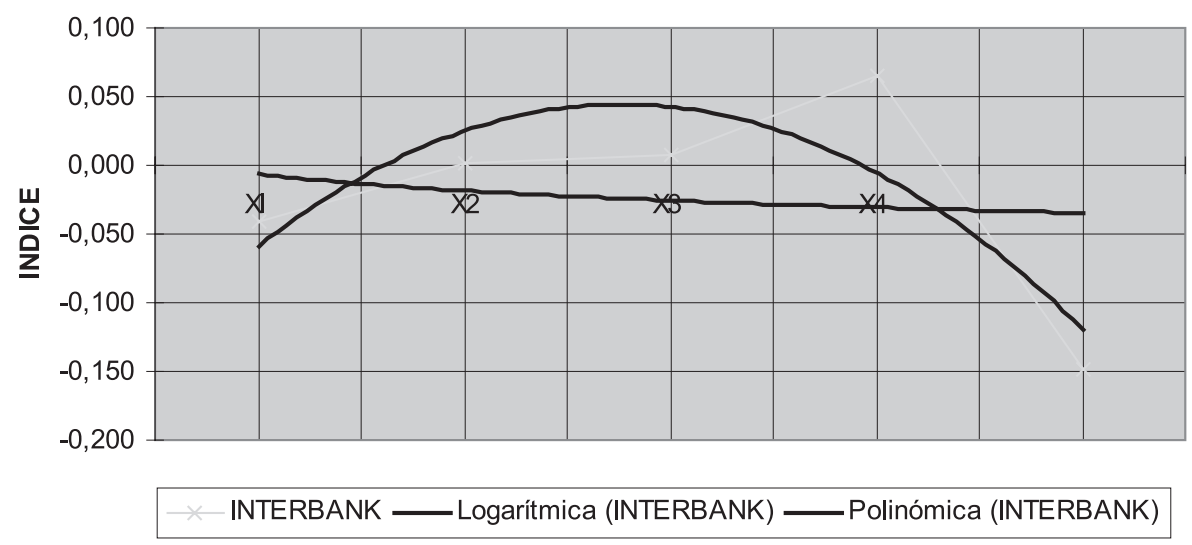

\section{Conclusiones}

Las instituciones financieras señaladas corren los riesgos que les son inherentes: falta de ejecución en tiempo; adquisición de bienes y servicios de baja calidad; adquisiciones innecesarias, inapropiadas y/o a precios elevados; falta de ejecución de una competencia plena y abierta.

Las presiones internas y externas que amenazan siempre la estabilidad financiera y la rentabilidad, también se suman con mayor rigor las operaciones irregulares y fraudulentas de personal de dirección que están relacionadas con decisiones ejecutivas. Esta ha sido la típica y constante causa de quiebra de las instituciones financieras. 
Bajo el rubro de integridad, se pueden examinar ocurrencias de soborno, divulgación no autorizada de información sensible, pagos ilegales, favoritismo, nepotismo, etc. Además, el clima de competencia entre los distintos instrumentos financieros por la iniciativa de ofrecer nuevas posibilidades a los clientes, para encontrar solución a los problemas de financiamiento, obliga a que las empresas definan su perfil de riesgo y le den seguimiento continuo para evitar la intromisión del virus de fracaso o quiebra.

Por lo tanto, deben participar todas las áreas funcionales en la definición del objetivo principal para entender y cuantificar los niveles de tolerancia y predecir la posible aparición del riesgo, que implique la alteración de la composición del negocio y, sobre todo, estar pendiente de que los flujos de caja esperados se mantengan conforme a lo planeado, no olvidando que la volatilidad puede hacer competitiva a una empresa hoy y echarla del mercado al día siguiente

En consecuencia, el problema no eestá en detectar los problemas que hacen que la gerencia falle y sus proyectos o planes queden truncados. El propósito es que los gerentes identifiquen los problemas para prevenir que incurran en una conducción errónea. Existe una frase en uso por los auditores: "información creíble pero errónea". Es decir: se trata de algo que parece que está bien, pero que ha perdido la precisión.

La gerencia debe estar alerta para captar las causas originales. No se puede corregir si no se llega a la fuente del problema. Los gerentes ya deben tener implementado un sistema apropiado para identificar debilidades operativas y realizar prueba de correcciones efectivas.

Si pueden tomar acción correctiva como gerentes, deben tomarla y si el asunto necesita proceder a un nivel más alto, deben llevarlo, para tomar la acción correctiva y luego deben convalidarla para ver si fue eficaz ó efectiva.

\section{Notas}

1 Ley 314- Ley general de bancos, instituciones financieras no bancarias y grupos financieros (Arto 47)

2 Explica con más detalle la administración del riesgo "Administración Financiera" de James C. Van Horne

3 Folleto de "Modulo de Finanzas Internacionales" (Made VII) página 13

4 La administración de riesgos: Una nueva tecnología, de la Gerencia Internacional BCN

\section{Referencias bibliográficas}

-BIBLIOTECA DEL BANCO CENTRAL, (1995). Elemento para un plan de acción en el evento de producirse la quiebra del Banades

-DIEZ DE CASTRO, L. y MASCARENAS, J., Ingeniería Financiera, segunda edición, Osborne/McGraw-Hill

-GERENCIA INTERNACIONAL BCN, (1996). La administración de riesgo: una nueva tecnología

-JORION, P., (1999). Valor en riesgo, Editorial Limusa 
-KRUGMAN, P. y OBSTFELD, M., Economía Internacional, tercera edición, Documentos -LA GACETA, DIARIO OFICIAL DE NICARAGUA, Ley $N^{\circ} 314$ del 18 de Octubre de 1999 -SUPERINTENDENCIA DE BANCOS DE NICARAGUA, (1995). Informe

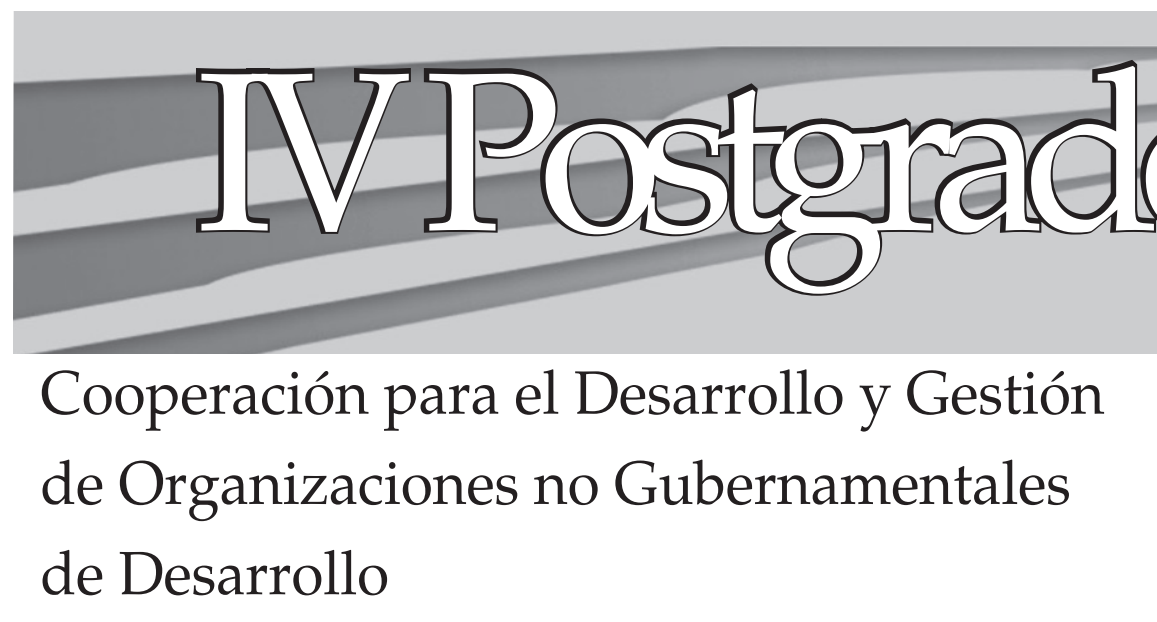

\section{Dirigido a}

Licenciados en Relaciones Internacionales, ciencias políticas, ciencias jurídicas, sociología, historia, economía, trabajo social. También pueden optar funcionarios de la administración pública en el campo de la cooperación y servicio exterior y gestores de la cooperación internacional.

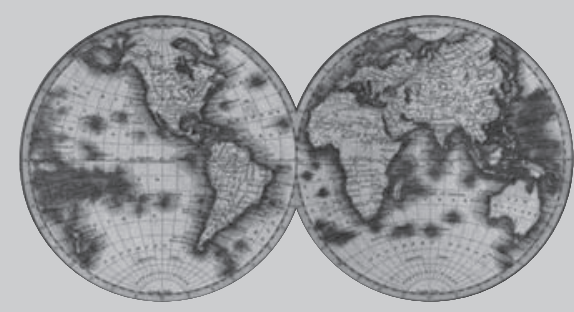

\section{Matrícula}

Julio 2006

\section{Inicio de clases}

24 de julio de 2006

\section{Horario}

De lunes a viernes de 6:00 a 9:00 p.m.

y eventualmente los sábados

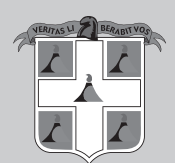

